\title{
Blood-Brain Barrier Disruption after Cardiac Surgery
}

\author{
J.G. Merino, L.L. Latour, A. Tso, K.Y. Lee, D.W. Kang, L.A. Davis, R.M. Lazar, K.A. Horvath, P.J. Corso, and S. Warach
}

t

\section{ABSTRACT}

BACKGROUND AND PURPOSE: CNS complications are often seen after heart surgery, and postsurgical disruption of the BBB may play an etiologic role. The objective of this study was to determine the prevalence of MR imaging-detected BBB disruption (HARM) and DWI lesions after cardiac surgery.

MATERIALS AND METHODS: All patients had an MRI after cardiac surgery. For half the patients (group 1), we administered gadolinium 24 hours after surgery and obtained high-resolution DWI and FLAIR images 24-48 hours later. We administered gadolinium to the other half (group 2) at the time of the postoperative scan, 2-4 days after surgery. Two stroke neurologists evaluated the images.

RESULTS: Of the 19 patients we studied, none had clinical evidence of a stroke or delirium at the time of the gadolinium administration or the scan, but 9 patients (47\%) had HARM (67\% in group 1; 30\% in group 2; $P=.18$ ) and 14 patients (74\%) had DWI lesions (70\% in group 1; $78 \%$ in group $2 ; P=1.0)$. Not all patients with DWI lesions had HARM, and not all patients with HARM had DWI lesions $(P=.56)$.

CONCLUSIONS: Almost half the patients undergoing cardiac surgery have evidence of HARM, and three-quarters have acute lesions on DWI after surgery. BBB disruption is more prevalent in the first 24 hours after surgery. These findings suggest that MR imaging can be used as an imaging biomarker to assess therapies that may protect the BBB in patients undergoing heart surgery.

ABBREVIATIONS: CABG $=$ coronary artery bypass grafting; HARM $=$ hyperintense acute reperfusion marker; $\mathrm{MMP}-9=$ matrix metalloproteinase-9; $\mathrm{mRS}=$ modified Rankin scale

B etween $1 \%$ and $6 \%$ of patients have clinical evidence of stroke after heart surgery, and the incidence of hyperintense lesions on DWI is even higher $(18 \%-60 \%)$. These DWI lesions typically are

Received February 23, 2012; accepted after revision June 16.

From the section on Stroke Diagnostics and Therapeutics (J.G.M., L.L.L., L.A.D., S.W.), National Institute of Neurological Disorders and Stroke, Bethesda, Maryland; Johns Hopkins Community Physicians (J.G.M., K.A.H.), Bethesda, Maryland; Department of Neurology (A.T.), University of California San Francisco, San Francisco, California; Department of Neurology (K.Y.L.), Yonsei University College of Medicine, Seoul, Republic of Korea; Asan Medical Center (D.W.K.), Seoul, Republic of Korea; Department of Neurology (R.M.L.), Columbia University College of Physicians and Surgeons, New York, New York; Cardiothoracic Surgery Research (K.A.H.), National Heart Lung and Blood Institute, Bethesda, Maryland; and Department of Cardiac Surgery (P.J.C.), Washington Hospital Center, Washington, DC.

This research was supported by the Division of Intramural Research of the $\mathrm{Na}$ tional Institute of Neurological Disorders and Stroke of the National Institutes of Health.

Paper previously presented in part at: 36th International Stroke Conference, February 9-11, 2011, Los Angeles, California.

Please address correspondence to José G. Merino, MD, Suburban Hospital Stroke Program, 8600 Old Georgetown Rd, Bethesda, MD 20814; e-mail: merinoj@ninds.nih.gov

- Indicates open access to non-subscribers at www.ajnr.org

三 Indicates article with supplemental on-line table.

http://dx.doi.org/10.3174/ajnr.A3251 small and often do not lead to clinical deficits, and the long-term clinical implications are not well understood. ${ }^{1-7}$ Because it leads to brain changes, cardiac surgery has been proposed as a model to screen potential neuroprotective agents, particularly when DWI is used to identify incident lesions. ${ }^{2,8}$

In addition to ischemia due to hypoperfusion and distal embolization, cardiac surgery elicits a systemic inflammatory response that may lead to a disruption of the BBB and neurologic dysfunction. ${ }^{9}$ In experimental models, the use of cardiopulmonary bypass leads to opening of the BBB. ${ }^{10,11}$ Disruption of the $\mathrm{BBB}$ can be seen on MR imaging as enhancement of the subarachnoid space on postcontrast FLAIR images. Under normal conditions, gadolinium-containing compounds do not cross the BBB. Under ischemic conditions, however, an increase in MMP-9 leads to proteolytic breakdown of the BBB integrity that allows passage of gadolinium to the CSF and the parenchyma. Because gadolinium shortens T1, it disrupts the CSF signal suppression of FLAIR, and CSF appears hyperintense. The sensitivity of FLAIR for lowcontrast concentrations is $>10$-fold higher than for T1WI, and much lower concentrations of gadolinium are required to see the gadolinium hyperintensity on FLAIR than on T1WI. ${ }^{12-16}$

The enhancement of the CSF on FLAIR, an imaging biomarker 
called HARM, is seen in $30 \%-40 \%$ of patients with acute stroke and approximately $20 \%$ of patients with TIA without DWI lesions on acute MR imaging; it is more common in patients treated with pharmacologic or mechanical reperfusion therapies. ${ }^{17,18}$ It has been observed in animal models of ischemia, in patients with acute stroke, and in patients undergoing reperfusion procedures, including carotid artery angioplasty and stent placement. ${ }^{12,13,17,19-22}$ It is associated with age, reperfusion, thrombolysis, endovascular procedures, changes in matrix metalloproteinases, hemorrhagic transformation, and poor outcome. ${ }^{17,20,21,23-26}$ Recent studies confirm that HARM is due to enhancement of the CSF and not of the parenchyma and that gadolinium in the CSF is the cause of the enhancement. ${ }^{15,20}$ HARM is a biomarker with potential use in proofof-concept clinical trials of agents that prevent BBB breakdown and consequent brain damage. ${ }^{27-29}$

The purpose of this study was to determine whether BBB disruption occurs after on- and off-pump CABG or valve repair surgery and, if so, its relationship to incident ischemic lesions.

\section{MATERIALS AND METHODS \\ Subjects}

We enrolled patients who had on- or off-pump CABG or aortic or mitral valve replacement if they were 18 years of age or older and functionally independent $(\mathrm{mRS}<2$ ). We excluded patients who had dementia, cognitive dysfunction, or a psychiatric disorder; were scheduled for concomitant carotid endarterectomy or pacemaker placement; had a contraindication to MR imaging; or were pregnant. The Institutional Review Board of the National Institute of Neurologic Disorders and Stroke and Suburban Hospital in Bethesda, Maryland, and Washington Hospital Center in Washington, DC, approved the study, and all patients gave informed consent to participate.

\section{Study Procedures}

Initially we enrolled patients in this study before surgery (patients in group 1). After several months, it became clear that it was not practical to enroll and receive consent from patients before surgery. Because the time between the decision to operate and the surgery was short, patients were reluctant to enroll in a study that required them to have a preoperative $M R$ imaging. We then started enrolling patients after surgery, once they were hemodynamically stable (group 2). Enrolling and imaging patients at different times allowed us to assess the permeability of the BBB at different time points. We administered gadolinium $(0.1 \mathrm{mmol} /$ $\mathrm{kg}$ ) to patients in group 1 approximately 24 hours after surgery; these patients had a postoperative MR imaging once they were hemodynamically stable (typically around 48 hours after surgery). The half-life of gadolinium in blood is 1.7 hours, yet it remains in the subarachnoid space for several days; any HARM on the postoperative MR imaging scan therefore reflects the status of the BBB around the time of the injection. Group 1 patients also had a preoperative MR imaging. Patients in group 2 received gadolinium at the time of the postoperative MR imaging and did not have a preoperative scan. Throughout the study, a neurologist examined the patients and administered the Mini-Mental State Examination and the Delirium Rating Scale before surgery and at the time of gadolinium administration (group 1) and at the time of the postoperative MR imaging scan (groups 1 and 2). ${ }^{30}$

\section{Imaging and Image Analysis}

Imaging was done in a 3T scanner (Philips Healthcare, Best, the Netherlands) or a 1.5T scanner (GE Healthcare, Milwaukee, Wisconsin); prior studies have shown that the field strength of the MR imaging does not affect the HARM detection rate. ${ }^{31}$ Typical MR imaging parameters include $24 \mathrm{~cm}$ FOV and 35 contiguous interleaved sections, 3.5-4-mm thick and colocalized across series; trace-weighted DWI images were obtained at $b=1000$ from a 13-15 direction DTI sequence with an in-plane resolution of $2.5 \times 2.5 \mathrm{~mm}$ and TR/TE $=10 \mathrm{~s} / 58 \mathrm{~ms}$ at $3 \mathrm{~T}$ or $10 \mathrm{~s} / 72 \mathrm{~ms}$ at $1.5 \mathrm{~T}$. T2-FLAIR images were obtained with an in-plane resolution of $0.94 \times 0.94 \mathrm{~mm}, \mathrm{TR} / \mathrm{TE}=9000 / 120 \mathrm{~ms}$ and $\mathrm{TI}=2600 \mathrm{~ms}$ at $3 \mathrm{~T}$ or $\mathrm{TR} / \mathrm{TE}=9000 / 140 \mathrm{~ms}$ and $\mathrm{TI}=2200 \mathrm{~ms}$ at $1.5 \mathrm{~T}$.

Two stroke neurologists blinded to the clinical and surgical variables read the MRI, and a third reader settled disagreements. The readers counted the number of lesions on the postoperative DWI and classified them on the basis of location (cortical, subcortical, or mixed) and appearance (punctate or confluent). They also reviewed the precontrast and postcontrast FLAIR images for the presence of HARM and classified it by location (sulcal, ventricular, and/or generalized) and severity (mild, moderate, or severe) (Fig 1).

\section{Statistical Analysis}

The primary outcome measures were evidence of postoperative ischemic lesions on DWI and presence of HARM on FLAIR. We analyzed the outcomes as dichotomous variables. We did the statistical analyses with SPSS for Mac (SPSS, Chicago, Illinois) and compared categoric variables by using $\chi^{2}$ or Fisher exact test and means with the independent sample $t$ test. All $P$ values are 2 -tailed.

\section{RESULTS}

We report the imaging findings in 19 patients with a mean age of 67.4 years $(S D \pm 0.2)$. Six patients had off-pump CABG and 13 had surgery with the use of a cardiopulmonary bypass pump (7 CABG and 6 valvular surgery). The mean pump time was 130 minutes ( $\mathrm{SD} \pm 23$; range, $82-179$ minutes), and the mean crossclamp time was 101 minutes ( $\mathrm{SD} \pm 27$, range, 56-160 minutes). The mean pump time was similar among the 7 patients who had CABG and the 6 who had valve repair $(141 \pm 22$ versus $120 \pm 22$ minutes, respectively; $P=.13$ ). Likewise, cross-clamp time did not differ between the patients who had CABG and those who had valve repair ( $109 \pm 26$ versus $92 \pm 26$ minutes, respectively; $P=$ .26). Seven patients were examined with the $3 \mathrm{~T}$ scanner and 12 with the $1.5 \mathrm{~T}$ scanner.

The mean time from surgery to gadolinium administration in patients in group 1 was $21.6 \pm 1.2$ hours; the corresponding value for patients in group 2 was $88.1 \pm 21.2$ hours $(P<.001)$. The integrity of the BBB was therefore assessed, on average, 21.6 hours after surgery in the first group of patients and 88.1 hours after surgery in the second group. The time from surgery to postoperative MR imaging was also shorter for group 1 than for group 2 (52 \pm 15.4 hours versus $88.1 \pm 21.2$ hours; $P<.001)$. The 9 patients in group 1 had a preoperative scan; only 1 (11\%) had 


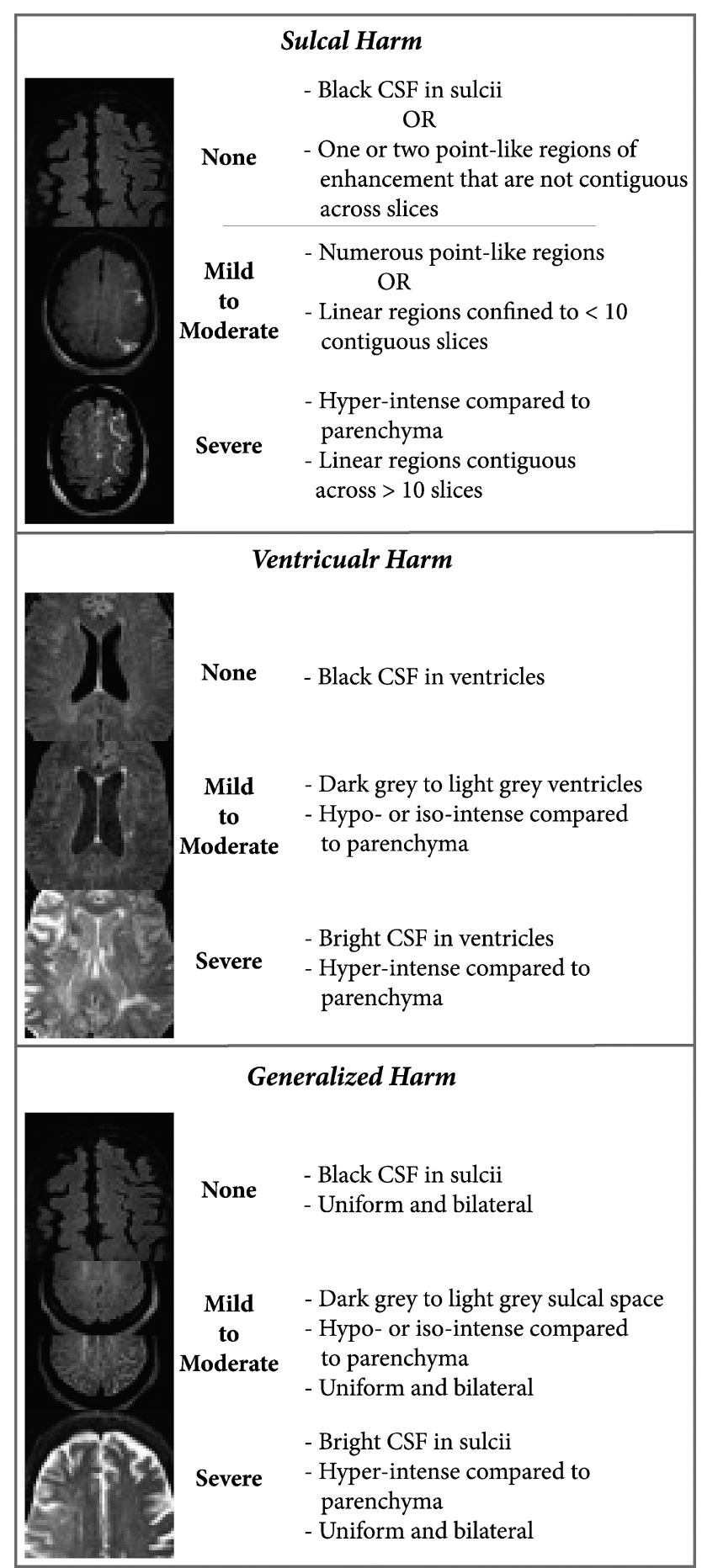

FIG 1. Classification of HARM by location and severity. Definitions used for HARM classification.

DWI lesions before surgery. None of the patients had clinical evidence of a stroke after surgery, and all had consistently normal neurologic examinations and scores on the Mini-Mental State Examination and Delirium Rating Scale.

We found HARM in 9 patients (47\%); of these, 7 (78\%) also had DWI lesions (Table). Most patients had mild/moderate HARM, and only 2 (both in group 1) had severe sulcal or background HARM (Figs 2 and 3). There were more than twice as many patients with HARM in group 1 than in group $2(66.7 \%$

\begin{tabular}{lccc} 
Association of HARM and DWI lesions & \\
\hline & \multicolumn{2}{c}{ HARM } & \\
\cline { 2 - 4 } & Yes & No & Total \\
\hline DWI Lesion & 7 & 7 & $14(74 \%)$ \\
No DWI Lesion & 2 & 3 & 5 \\
Total & $9(47 \%)$ & 10 & 19 \\
\hline
\end{tabular}

versus $30 \% ; P=.18)$. The proportion of patients with HARM was similar in patients who had on- versus off-pump procedures $(38 \%$ versus $50 \% ; P=1.0)$.

DWI lesions were seen in 14 (74\%) of the 19 patients who had a postoperative scan. In these patients, the mean number of lesions was $5.6 \pm 4.7$ and the median was 4 (range, 1-18). The proportion of patients with DWI lesions was similar in groups 1 and $2(78 \%$ versus $70 \% ; P=1.0)$. The incidence of lesions (as a dichotomous variable) did not differ by type of surgery (valvular, CABG, and off-pump CABG; $P=.54)$. The proportion of patients who had DWI lesions was similar ( $83 \%$ versus $69 \% ; P=.63$ ) in those who had off-pump versus on-pump procedures. Not all patients with DWI lesions had HARM, and not all patients with HARM had DWI lesions, and the association between DWI lesions and HARM was not significant $(P=.56$; Table 1$)$. Among the 7 patients who had DWI lesions and HARM, 3 had background HARM only; 1 had sulcal, background, and ventricular HARM; and 3 had sulcal HARM only. The 4 patients with sulcal HARM had DWI lesions in multiple vascular territories. In 2 of these patients, the HARM was not in the vascular territory of the DWI lesions. In the other 2 patients, there was HARM in at least 1 of the affected vascular territories, but not all sulcal HARM was contiguous with the DWI lesion (On-line Table).

\section{DISCUSSION}

MR imaging demonstrated that BBB disruption after cardiac surgery occurred in almost half the patients in our sample. The incidence of HARM was higher among patients who received gadolinium within 24 hours of surgery. Because of the short plasma distribution ( $0.2 \pm 0.13$ hours) and elimination half-life (1.6 \pm 0.13 hours) of gadolinium, our findings suggest that the BBB may open during or soon after heart surgery and close shortly thereafter. Animal studies show that the BBB opens soon after ischemia. In an MCA occlusion rat model, brain sucrose uptake, a marker of BBB disruption, increased 3 hours after reperfusion, was maximal at 48 hours, and persisted for up to 14 days. ${ }^{32}$ In another study, gadolinium enhancement of the ventricles ipsilateral to stroke was seen on FLAIR within 1 hour of reperfusion in rats subject to MCA occlusion, suggesting that the blood-CSF barrier can be disrupted within minutes of onset of ischemia, and this enhancement increases by 24 hours. At 48 hours, parenchymal enhancement on T1 was evident, suggesting a more widespread BBB opening. ${ }^{23}$ In humans with stroke, HARM can be seen within the first 6 hours after the onset of symptoms and persists for up to 5 days. $^{17}$

The clinical relevance of HARM in patients undergoing cardiac surgery is unclear, because our patients did not have evidence of neurologic or cognitive dysfunction at the time of gadolinium administration-when the integrity of the BBB was evaluated-or at the time of the MR imaging scan. Further studies are 


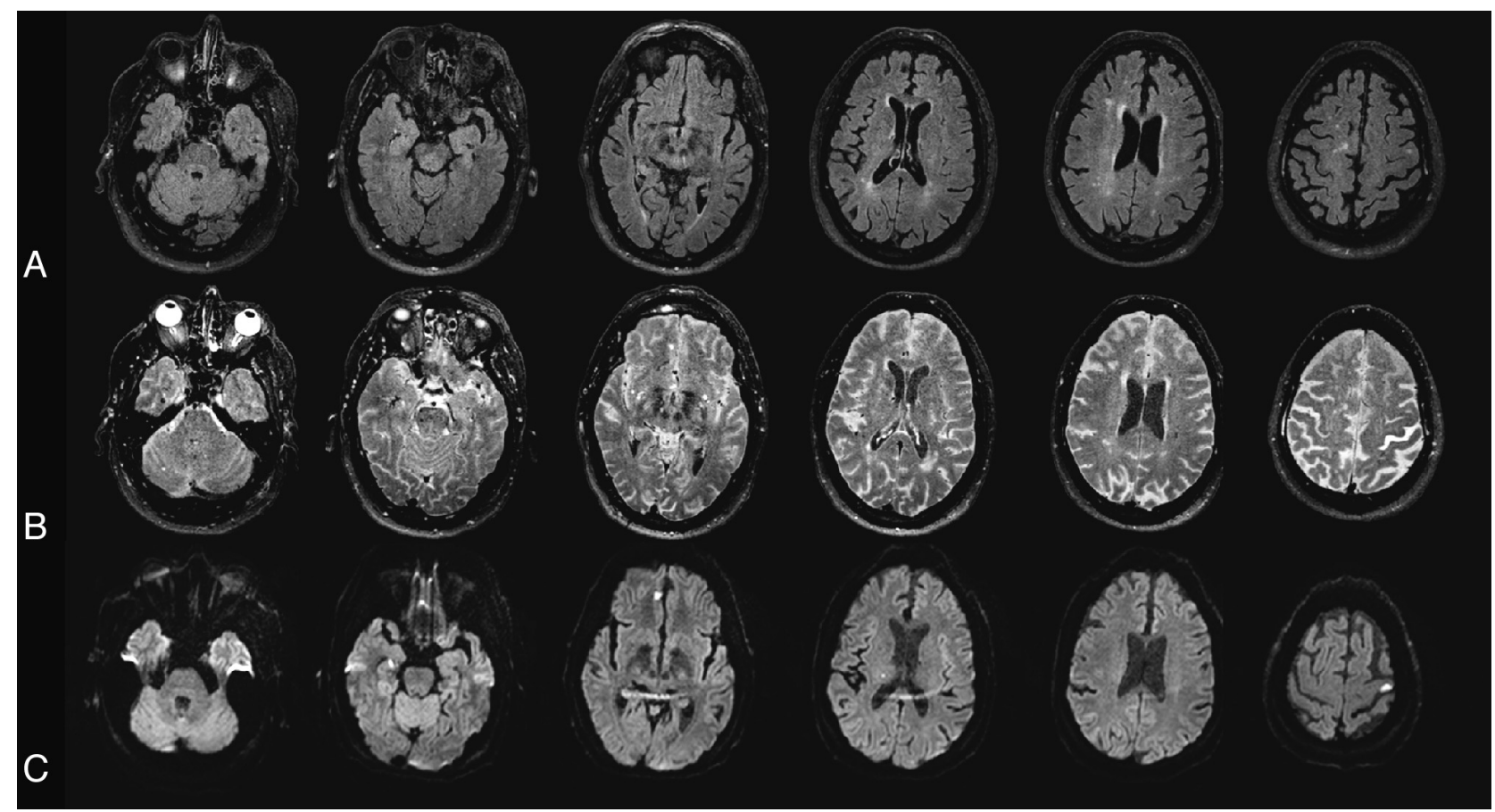

FIG 2. A 74-year-old man who underwent off-pump CABG. A, Baseline FLAIR obtained before surgery. B, Postgadolinium scan. Gadolinium was administered 20 hours after surgery, and 3T MR imaging was performed 20 hours later. Postgadolinium FLAIR shows enhancement throughout the subarachnoid space (severe sulcal and background HARM). The CSF in the ventricles appears gray (mild/moderate ventricular HARM). Gadolinium enhancement is also seen in the eye. C, DWI obtained after surgery. Patient had 4 DWI lesions.

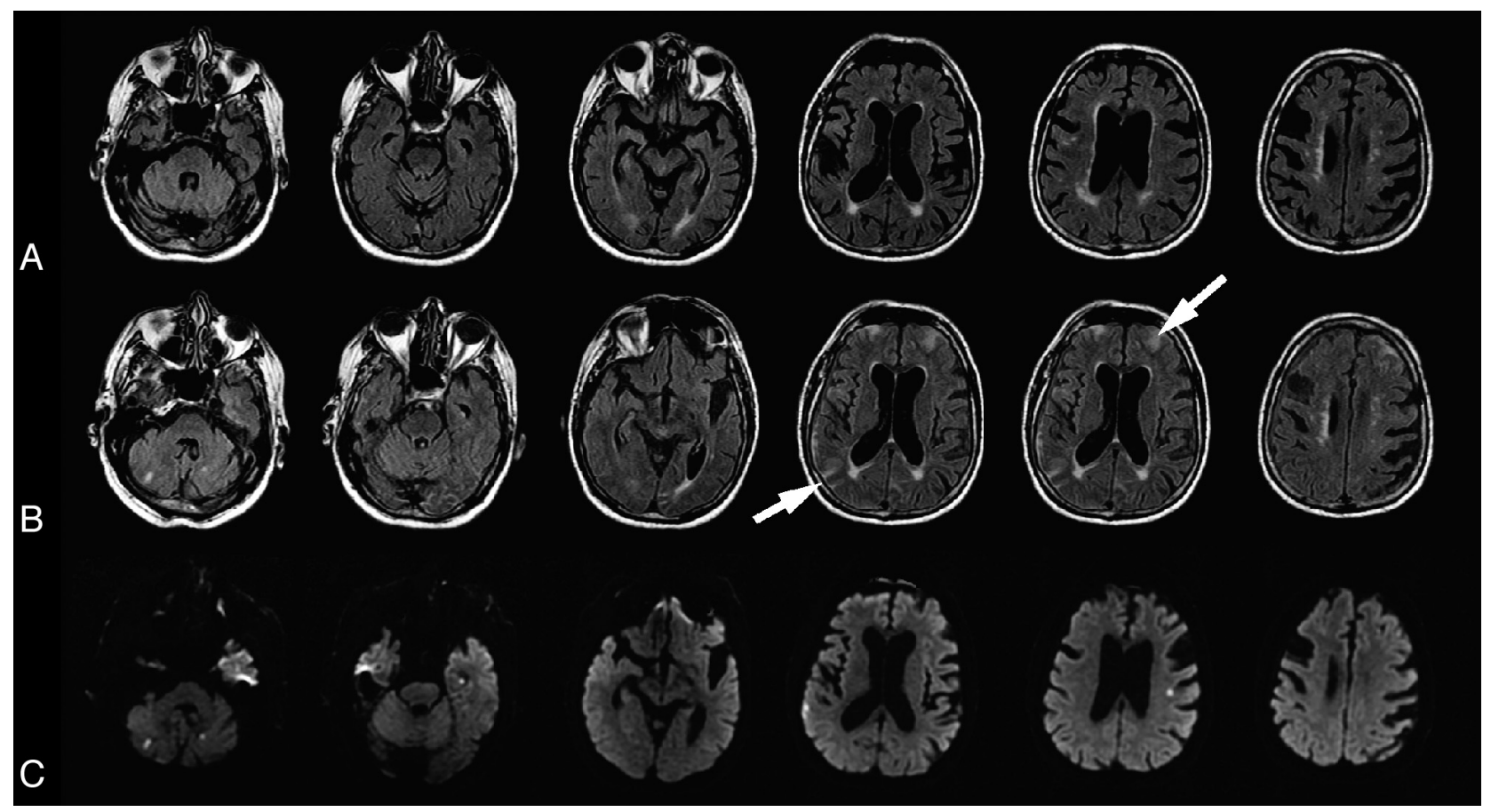

FIG 3. An 86-year-old man who underwent CABG. $A$, Baseline FLAIR performed before surgery. $B$, Postgadolinium FLAIR. Gadolinium was administered 23 hours after surgery, and the 1.5T MR imaging was obtained 27 hours later. Postgadolinium FLAIR shows bright enhancement in the right frontal and left parietal subarachnoid space (arrows, severe sulcal HARM). C, Postoperative DWI. The patient had 6 DWI lesions.

needed to determine whether BBB disruption after heart surgery is clinically relevant and if it occurs because of focal or global ischemia, reperfusion injury, changes in the level of matrix metalloproteinases, or inflammatory mechanisms. Our findings sug- gest that such studies should focus on the first 24 hours after surgery.

The incidence of DWI lesions in our series is higher than previously reported. ${ }^{1-7}$ Several features of our MR imaging sequences

AJNR Am J Neuroradiol 34:518-23 Mar 2013 www.ajnr.org 
may explain this higher incidence: we used 1) thin sections to decrease partial volume averaging with normal tissue on CSF; 2) short TE to decrease T2 loss; 3) a DTI sequence with 13-15 directions instead of a conventional DWI sequence to increase averaging and signal intensity-to-noise ratio; and, 4) on the $1.5 \mathrm{~T}$ scanner, a twice refocused spin-echo to decrease eddy currents.

In our patients, most DWI lesions were cortical. The clinical relevance of such small DWI lesions seen after invasive procedures, however, remains unknown. ${ }^{33}$ Case studies have shown that, over time, some of these lesions may become invisible on 3D T1WI and FLAIR images but may still lead to a loss of gray matter. ${ }^{34}$ These small cortical lesions may be another pathway to cortical atrophy and may explain why some patients undergoing cardiac surgery develop cognitive impairment.

In our study, we found no association between HARM and DWI lesions. Cerebral ischemia and reperfusion are associated with opening of the $\mathrm{BBB}$, which has been described in patients with transient symptoms without DWI lesions. ${ }^{18}$ A proposed mechanism for BBB disruption after ischemia of any duration is activation of inflammatory cascades and proteolytic enzymes. ${ }^{25,35}$ The effect of ischemia on $\mathrm{BBB}$ disruption may be diffuse. ${ }^{26}$ HARM is not always related to the site of ischemia. ${ }^{17,21}$ In addition, in patients undergoing cardiac surgery, the procedure activates a systemic inflammatory response and the production of immune mediators that may contribute to the development of acute and chronic neurologic dysfunction and disruption of the $\mathrm{BBB}$, a process also seen in patients with traumatic brain injury and hemorrhagic and ischemic stroke. ${ }^{36,37}$ Future studies are needed to clarify the role of different immune mediators in the genesis of HARM in these patients.

The use of cardiopulmonary bypass may lead to disruption of the $\mathrm{BBB}$, perhaps on the basis of a systemic inflammatory response. ${ }^{9,11}$ Case series suggest that neurologic complications are fewer in off-pump CABG, but clinical trials have not confirmed this observation. ${ }^{38,39}$ In our series, the proportion of patients with DWI lesions and HARM was similar in patients who had an offpump CABG and those who had an on-pump procedure (CABG or valve replacement). Why disruption of the BBB occurs in patients who have an off-pump procedure is not clear and merits further study of serum biomarkers of inflammation and levels of different matrix metalloproteinases.

Our study has some limitations; thus, our findings are preliminary and require replication in a larger prospective study. Our sample was small, and the lack of association between HARM and DWI lesions may be a type II error rather than a definitive finding. It is possible that some of the DWI lesions were present before surgery, but only 1 of the 9 participants who had a presurgical MR imaging had DWI lesions at baseline. Breathing a high oxygen concentration may lead to CSF hyperintensity on FLAIR because of the T1-shortening effect of oxygen, a phenomenon described only in intubated patients and volunteers breathing $100 \% \mathrm{O}_{2}$; in that case, the CSF enhancement is global and severe. ${ }^{40,41}$ We do not have information about the use of supplemental oxygen in our patients, but we know that none was intubated or used a nonrebreather mask, so we assume that any HARM was not due to high oxygen concentration in the CSF. Patients were subject to a variety of surgical procedures, but because of the sample size, we could not determine whether features inherent to each surgery played an etiologic role in the development of brain changes. Finally, we do not have longitudinal follow-up data to determine the clinical implications of BBB disruption shortly after cardiac surgery.

\section{CONCLUSIONS}

Almost half the patients undergoing cardiac surgery have evidence of HARM, three-quarters of the patients have acute lesions on DWI after surgery, and BBB disruption is more prevalent in the first 24 hours after surgery. These findings suggest that MR imaging can be used as an imaging biomarker to assess therapies that may protect the BBB in patients undergoing heart surgery.

\section{ACKNOWLEDGMENTS}

We would like to acknowledge the assistance of the Peter C. Hill, $\mathrm{MD}$, and the cardiac surgery and National Institutes of Health Stroke Teams at Suburban Hospital and Washington Hospital Center in the performance of this study.

Disclosures: José Merino—UNRELATED: Employment: Johns Hopkins Community Physicians, NINDS Intramural Division, Comments: I am employed by Johns Hopkins Community Physicians. Part of my salary is paid by the NINDS Intramural Division through a contract between the National Institutes of Health and Suburban Hospital; American Academy of Neurology, Fred Hutchinson Research Institute, University of British Columbia, WebMD, Comments: 1. I was paid an honorarium for my role as Associate Editor of AAN.com, the Web site of the American Academy of Neurology. 2. I was paid for my role as stroke outcome adjudicator on a case-by-case basis for the Women's Health Initiative and the SPS3 study. The National Institutes of Health funds both studies. The payment came from the Fred Hutchinson Research Institute and the UBC. 3. I was paid an honorarium for doing a Web interview for WebMD. Ronald Lazar-RELATED: Grant: National Institutes of Health, Comments: Extramural consultant.

\section{REFERENCES}

1. Bendszus M, Reents W, Franke D, et al. Brain damage after coronary artery bypass grafting. Arch Neurol 2002;59:1090-95

2. Restrepo L, Wityk RJ, Grega MA, et al. Diffusion- and perfusionweighted magnetic resonance imaging of the brain before and after coronary artery bypass grafting surgery. Stroke 2002;33:2909-15

3. Knipp SC, Matatko N, Wilhelm H, et al. Evaluation of brain injury after coronary artery bypass grafting. A prospective study using neuropsychological assessment and diffusion-weighted magnetic resonance imaging. Eur J Cardiothorac Surg 2004;25:791-800

4. Djaiani G, Fedorko L, Borger M, et al. Mild to moderate atheromatous disease of the thoracic aorta and new ischemic brain lesions after conventional coronary artery bypass graft surgery. Stroke 2004;35:e356-58

5. Floyd TF, Shah PN, Price CC, et al. Clinically silent cerebral ischemic events after cardiac surgery: their incidence, regional vascular occurrence, and procedural dependence. Ann Thorac Surg 2006;81: $2160-66$

6. Knipp SC, Matatko N, Schlamann M, et al. Small ischemic brain lesions after cardiac valve replacement detected by diffusionweighted magnetic resonance imaging: relation to neurocognitive function. Eur J Cardiothorac Surg 2005;28:88-96

7. Jeon SB, Lee JW, Kim SJ, et al. New cerebral lesions on T2*-weighted gradient-echo imaging after cardiac valve surgery. Cerebrovasc Dis 2010;30:194-99

8. Grieco G, d'Hollosy M, Culliford AT, et al. Evaluating neuroprotective agents for clinical anti-ischemic benefit using neurological and neuropsychological changes after cardiac surgery under cardiopulmonary bypass. Methodological strategies and results of a doubleblind, placebo-controlled trial of GM1 ganglioside. Stroke 1996;27: $858-74$ 
9. Rinder C. Cellular inflammatory response and clinical outcome in cardiac surgery. Curr Opin Anaesthesiol 2006;19:65-68

10. Cavaglia M, Seshadri SG, Marchand JE, et al. Increased transcription factor expression and permeability of the blood brain barrier associated with cardiopulmonary bypass in lambs. Ann Thorac Surg 2004;78:1418-25

11. Okamura T, Ishibashi N, Zurakowski D, et al. Cardiopulmonary bypass increases permeability of the blood-cerebrospinal fluid barrier. Ann Thorac Surg 2010;89:187-94

12. Wilkinson ID, Griffiths PD, Hoggard N, et al. Unilateral leptomeningeal enhancement after carotid stent insertion detected by magnetic resonance imaging. Stroke 2000;31:848-51

13. Dechambre SD, Duprez T, Grandin CB, et al. High signal in cerebrospinal fluid mimicking subarachnoid haemorrhage on FLAIR following acute stroke and intravenous contrast medium. Neuroradiology 2000;42:608-11

14. Mamourian AC, Hoopes PJ, Lewis LD. Visualization of intravenously administered contrast material in the CSF on fluid-attenuated inversion-recovery MR images: an in vitro and animal-model investigation. AJNR Am J Neuroradiol 2000;21:105-11

15. Köhrmann M, Struffert T, Frenzel T, et al. The hyperintense acute reperfusion marker on fluid-attenuated inversion recovery magnetic resonance imaging is caused by gadolinium in the cerebrospinal fluid. Stroke 2012;43:259-61

16. Mathews VP, Caldemeyer KS, Lowe MJ, et al. Brain: gadoliniumenhanced fast fluid-attenuated inversion-recovery MR imaging. Radiology 1999;211:257-63

17. Latour LL, Kang DW, Ezzeddine MA, et al. Early blood-brain barrier disruption in human focal brain ischemia. Ann Neurol 2004;56: $468-77$

18. Rozanski M, Ebinger M, Schmidt WU, et al. Hyperintense acute reperfusion marker on FLAIR is not associated with early haemorrhagic transformation in the elderly. Eur Radiol 2010;20:2990-96

19. Henning EC, Latour LL, Hallenbeck JM, et al. Reperfusion-associated hemorrhagic transformation in SHR rats: evidence of symptomatic parenchymal hematoma. Stroke 2008;39:3405-10

20. Henning EC, Latour LL, Warach S. Verification of enhancement of the CSF space, not parenchyma, in acute stroke patients with early blood-brain barrier disruption. J Cereb Blood Flow Metab 2008;28: $882-86$

21. Kidwell CS, Latour L, Saver JL, et al. Thrombolytic toxicity: blood brain barrier disruption in human ischemic stroke. Cerebrovasc Dis 2008;25:338-43

22. Michel E, Liu H, Remley KB, et al. Perfusion MR neuroimaging in patients undergoing balloon test occlusion of the internal carotid artery. AJNR Am J Neuroradiol 2001;22:1590-96

23. Batra A, Latour LL, Ruetzler CA, et al. Increased plasma and tissue MMP levels are associated with BCSFB and BBB disruption evident on post-contrast FLAIR after experimental stroke. J Cereb Blood Flow Metab 2010;30:1188-99

24. Warach S, Latour LL. Evidence of reperfusion injury, exacerbated by thrombolytic therapy, in human focal brain ischemia using a novel imaging marker of early blood-brain barrier disruption. Stroke 2004;35:2659-61

25. Barr TL, Latour LL, Lee KY, et al. Blood-brain barrier disruption in humans is independently associated with increased matrix metalloproteinase-9. Stroke 2010;41:e123-28

26. Wardlaw JM, Doubal F, Armitage P, et al. Lacunar stroke is associated with diffuse blood-brain barrier dysfunction. Ann Neurol 2009; 65:194-202

27. Murata Y, Rosell A, Scannevin RH, et al. Extension of the thrombolytic time window with minocycline in experimental stroke. Stroke 2008;39:3372-77

28. Sood RR, Taheri S, Candelario-Jalil E, et al. Early beneficial effect of matrix metalloproteinase inhibition on blood-brain barrier permeability as measured by magnetic resonance imaging countered by impaired long-term recovery after stroke in rat brain. $J$ Cereb Blood Flow Metab 2008;28:431-38

29. Nagel S, Su Y, Horstmann S, et al. Minocycline and hypothermia for reperfusion injury after focal cerebral ischemia in the rat: effects on BBB breakdown and MMP expression in the acute and subacute phase. Brain Res 2008;1188:198-206

30. Trzepacz PT, Baker RW, Greenhouse J. A symptom rating scale for delirium. Psychiatry Res 1988;23:89-97

31. Batra A, Song SS, Merino JG, et al. Older patients are at higher risk for blood-brain barrier disruption following treatment with IVTPA, independent of impaired kidney function. Stroke 2011;42:e253

32. Rosenberg GA, Estrada EY, Dencoff JE. Matrix metalloproteinases and TIMPS are associated with blood-brain barrier opening after reperfusion in rat brain. Stroke 1998;29:2189-95

33. Altinbas A, van Zandvoort MJ, van den Berg E, et al. Cognition after carotid endarterectomy or stenting: a randomized comparison. Neurology 2011;77:1084-90

34. Sheng X, Thomas A, Warach S, et al. Acute cortical lesions "disappear" on MRI but are associated with cortical atrophy in stroke patients: implications for brain aging and vascular cognitive impairment. Stroke 2010;41:e14

35. Alvarez-Sabín J, Delgado P, Abilleira S, et al. Temporal profile of matrix metalloproteinases and their inhibitors after spontaneous intracerebral hemorrhage: relationship to clinical and radiological outcome. Stroke 2004;35:1316-22

36. van Harten AE, Scheeren TW, Absalom AR. A review of postoperative cognitive dysfunction and neuroinflammation associated with cardiac surgery and anaesthesia. Anaesthesia 2012;67:280-93

37. Shlosberg D, Benifla M, Kaufer D, et al. Blood-brain barrier breakdown as a therapeutic target in traumatic brain injury. Nat Rev Neurol 2010;6:393-403

38. Biancari F, Mosorin M, Rasinaho E, et al. Postoperative stroke after off-pump versus on-pump coronary artery bypass surgery. J Thorac Cardiovasc Surg 2007;133:169-73

39. van Dijk D, Spoor M, Hijman R, et al. Cognitive and cardiac outcomes $\mathbf{5}$ years after off-pump vs on-pump coronary artery bypass graft surgery. JAMA 2007;297:701-08

40. Deliganis AV, Fisher DJ, Lam AM, et al. Cerebrospinal fluid signal intensity increase on FLAIR MR images in patients under general anesthesia: the role of supplemental $\mathbf{O}_{2}$. Radiology 2001;218:152-56

41. Anzai Y, Ishikawa M, Shaw DW, et al. Paramagnetic effect of supplemental oxygen on CSF hyperintensity on fluid-attenuated inversion recovery MR images. AJNR Am J Neuroradiol 2004;25:274-79 